Ferrata Storti Foundation

\title{
The ASXL1-G643W variant accelerates the development of CEBPA mutant acute myeloid leukemia
}

\author{
Teresa D'Altri, ${ }^{1,2,3}$ Anna S. Wilhelmson, ${ }^{1,2,3}$ Mikkel B. Schuster, ${ }^{1,2,3}$ \\ Anne Wenzel, ${ }^{1,2,3}$ Adrija Kalvisa, ${ }^{1,2,3}$ Sachin Pundhir,, \\ Anne Meldgaard Hansen ${ }^{1,2,3}$ and Bo T. Porse ${ }^{1,2,3}$
}

Haematologica 2021

Volume 106(4):1000-1007

\section{Correspondence:}

BO PORSE

bo.porse@finsenlab.dk

Received: August 16, 2019.

Accepted: March 19, 2020.

Pre-published: May 7, 2020.

https://doi.org/10.3324/haematol.2019.235150

(C)2021 Ferrata Storti Foundation

Material published in Haematologica is covered by copyright. All rights are reserved to the Ferrata Storti Foundation. Use of published material is allowed under the following terms and conditions:

https://creativecommons.org/licenses/by-nc/4.0/legalcode. Copies of published material are allowed for personal or internal use. Sharing published material for non-commercial purposes is subject to the following conditions:

https://creativecommons. oró/licenses/by-nc/4.0/legalcode, sect. 3. Reproducing and sharing published material for commercial purposes is not allowed without permission in writing from the publisher.

${ }^{1}$ The Finsen Laboratory, Rigshospitalet, Faculty of Health Sciences, University of Copenhagen; ${ }^{2}$ Biotech Research and Innovation Centre (BRIC), University of Copenhagen and ${ }^{3}$ Danish Stem Cell Center (DanStem) Faculty of Health Sciences, University of Copenhagen, Copenhagen, Denmark

\section{ABSTRACT}

$\Lambda$ SXL1 is one of the most commonly mutated genes in myeloid malignancies, including myelodysplastic syndrome (MDS) and acute myeloid leukemia (AML). In order to further our understanding of the role of ASXL1 lesions in malignant hematopoiesis, we generated a novel knockin mouse model carrying the most frequent ASXL1 mutation identified in MDS patients, ASXL1 p.G643WfsX12. Mutant mice neither displayed any major hematopoietic defects nor developed any apparent hematological disease. In AML patients, ASXL1 mutations co-occur with mutations in CEBPA and we therefore generated compound Cebpa and Asxl1 mutated mice. Using a transplantation model, we found that the mutated Asxl1 allele significantly accelerated disease development in a CEBPA mutant context. Importantly, we demonstrated that, similar to the human setting, Asxll mutated mice responded poorly to chemotherapy. This model therefore constitutes an excellent experimental system for further studies into the clinically important question of chemotherapy resistance mediated by mutant ASXI1.

\section{Introduction}

Additional sex comb-like 1 (ASXL1) is a frequently mutated gene in myeloid malignancies including myelodysplastic syndrome (MDS) and acute myeloid leukemia (AML). ${ }^{1,2}$ Moreover, ASXL1 mutations are also highly prevalent in premalignant states such as clonal hematopoiesis of indeterminate potential (CHIP), demonstrating that ASXL1 lesions are early driver events with the potential to predispose for further malignant transformation. ${ }^{3,4}$ The vast majority of ASXL1 mutations are located in the last exon and are deletions, insertions, or substitutions resulting in stop codon mutations and truncation of the ASXL1 protein..$^{1,2}$ Mutations are always monoallelic and mRNA expression levels are variable. Due to difficulties in detecting ASXL1 in human samples, ASXL1 mutations were originally believed to be loss of function lesions and consequently haploinsufficient. ${ }^{5}$ However, in a more recent work, the truncated protein can indeed be detected, raising the possibility that ASXL1 mutations may act as dominant negative or gain of function variants. ${ }^{6}$

Mechanistically, ASXL1 is a dual function epigenetic regulator. Specifically, it interacts directly with the BRCA1-associated protein 1 (BAP1) to form a complex which de-ubiquitinates H2AK119Ub, a repressive histone mark deposited by the polycomb repressive complex 1 (PRC1). ${ }^{5,7,8}$ Moreover, ASXL1 interacts with the components of the polycomb repressive complex 2 (PRC2) which deposits the H3K27me3 repressive mark. ${ }^{5,9}$

The interest in the role of ASXL1 in malignant hematopoiesis has spurred the development of several Asxll knockout mouse models ${ }^{10-12}$ (for a recent review, see ${ }^{13}$ ) Although these models do not result in identical phenotypes, they all show phenotypes consistent with human MDS. Interestingly, overexpression of a truncated form of Asxll yielded similar results suggesting that the mutations found in patients 
result in a dominant-negative version of the protein. ${ }^{6}$ In line with these findings, several reports have indicated that truncated ASXL1 enhances BAP1 complex activity, thereby promoting depletion of the H2AK119Ub mark and aberrant myeloid differentiation. ${ }^{14,15}$ More refined modeling of ASXL1 function in hematological malignancies has been performed by knockin of patient-specific ASXL1 lesions into the murine Asxl1 locus. ${ }^{16,17}$ These knockin mice generally exhibit more subtle phenotypes compared to the complete deletion of the gene. Although heterozygous knockin mice do no develop MDS or $\mathrm{AML}$, the knockin alleles collaborate with other leukemic drivers such as MN1 and RUNX1 or accelerate AML development in an insertional mutagenesis setting. ${ }^{16,17}$

CEBPA is a key myeloid transcription factor which is mutated in approximately $10 \%$ of AML patients and biallelic CEBPA mutant AML constitutes a specific AML subtype. ${ }^{18-20}$ These patients either harbor biallelic N-terminal lesions or, more frequently, combine these lesions with a $\mathrm{C}$-terminal mutation. Whereas the $\mathrm{N}$-terminal lesions promote the expression of the $\mathrm{N}$-terminally truncated p30 isoform, C-terminal mutations result in variants that are unable to dimerize and are consequently inactive. Hence, the genetic lesions in biallelic CEBPA mutant AML converge at the expression of the $\mathrm{N}$-terminally truncated p30 isoform in the form of CEBPA-p30 homodimers. ${ }^{20}$ In stark contrast to full-length CEBPA, CEBPA-p30 is not able to repress E2F-mediated cell cycle progression ${ }^{21}$ and recent work has also identified specific downstream targets of this oncogenic CEBPA variant. ${ }^{22}$ Importantly, mice in which CEBPA-p30 expression is driven from the endogenous Cebpa locus develop AML within the first year of their lives. ${ }^{21}$ Interestingly, mutations in ASXL1 are frequent in biallelic CEBPA mutant AML, but how these two sets of lesions interact functionally is currently unknown. ${ }^{23,24}$

In the present work, we generated a novel Asxll knockin line by introducing the most common diseaseassociated mutation (p.G643WfsX12) into the murine Asxll allele. ${ }^{2,25}$ In order to assess the importance of Asxll lesions in the context of biallelic CEBPA mutated AML, we combined lesions in these two proteins and found that the ASXI1 mutation accelerated the development of CEBPA-p30 driven AML. Gene expression analysis yielded potential drivers of the accelerated phenotype. Interestingly, ASXL1 mutated AML were largely refractory to chemotherapy, thereby paralleling the findings from the human setting.

\section{Methods}

\section{Generation of the Asxl1 ${ }^{6643 W}$ knockin mice}

The Asxl1 G $G^{643 W}$ knockin line was generated using the double nicking CRISPR-Cas9 system in embryonic stem cells (ESC), followed by blastocyst injection. Two pspCas9n-2A-Puro constructs containing the two Asxl1 target sequences were electroporated into C57BL/6N ESCs together with a 141-mer single stranded (ss) DNA correction template containing the desired mutation. ESC clones were screened for the presence of the mutation and correctly targeted clones were injected into mouse blastocysts. Please refer to the Online Supplementary Appendix for additional details.

F1 offspring were backcrossed into C57BL/6 and maintained on that background. Animals were housed in a specific-pathogen-free facility and all procedures were approved by the Danish Animal Ethical Committee.

\section{In vivo acute myeloid leukemia development}

Bone marrow (BM) cells were retrieved and frozen in fetal calf serum (FCS) with 10\% DMSO. For leukemic experiments, stored BM was thawed and 2 million viable cells were transplanted into lethally irradiated (900 Gy) recipients by tail vein injection. Three weeks later, recipients were subjected to three intraperitoneal injections with poly-IC $(0.3 \mathrm{mg}$ in $200 \mu \mathrm{L}$ PBS, GE Healthcare) separated by 48 hours. Recipient mice were monitored for leukemic development and euthanized when moribund. Please refer to the Online Supplementary Appendix for additional details.

For the chemotherapy experiments, we transplanted cohorts of sublethally irradiated recipients with frozen secondary AML. Three weeks after transplant half the mice in each cohort were treated for 3 days with cytarabine $50 \mathrm{mg} / \mathrm{kg}$ and doxorubicine 1 $\mathrm{mg} / \mathrm{kg}$ and for 2 days with cytarabine $50 \mathrm{mg} / \mathrm{kg}$. The remaining mice received PBS as vehicle treatment. 25-30 $\mu \mathrm{L}$ of blood were harvested for analysis three days after the last injection. Leukemic cell numbers were determined by combining cell counting with CD45.1 (recipient)/ CD45.2 (donor) flow cytometry. For the survival study, the mice were observed for signs of disease and euthanized when moribund.

\section{Flow cytometry analysis and cell sorting}

For blood analysis, $50 \mu \mathrm{L}$ blood was collected from the facial vein. Erythrocytes were depleted with BD PharmLyse. For BM analysis, cells were collected by crushing tibia, femur and ilium and filtered. Blood or BM nucleated cells were washed in PBS with $3 \%$ FCS and stained for $15 \mathrm{~min}$ at $4^{\circ} \mathrm{C}$. Please refer to the Online Supplementary Appendix for additional details (antibodies and marker combinations).

\section{Statistics}

Unpaired t-test was used to compare values in the different groups. Log-rank (Mantel-Cox) test was used to compare survival distributions. For the chemotherapy data in Figure $5 \mathrm{~B}$ a one-tailed Mann-Whitney U test was used.

\section{RNA sequencing}

Donor derived AML blasts (CD45.2, Ter119-, B220-, CD3-, Mac1low, Gr1low, c-Kit ${ }^{+}$) were sorted from frozen BM samples into RLT lysis buffer (Qiagen) and RNA was extracted using the RNA Microkit (Qiagen). 100 ng RNA was used for the library generation, using TruSeq-V2 kit (Illumina). The libraries were analyzed by Obit (ThermoFisher) and Bioanalyzer (Agilent) and pooled in equimolar amounts. Multiplexed samples were sequenced on a NextSeq 500 (Illumina) yielding approximately 35-45 million reads per sample. Please refer to the Online Supplementary Appendix for additional details.

\section{Results}

\section{Generation of the Asxl1 $1^{6643 \mathrm{~W}}$ knockin mouse line}

In order to model the role of ASXL1 lesions in hematopoietic malignancies in the best possible manner, we decided to generate a mouse line expressing the most common ASXI1 mutation (G643WfsX12, from hereon G643W) found in MDS patients. ${ }^{2,25}$ Specifically, we used a double nicking CRISPR-Cas9 system in combination with a $141 \mathrm{bp}$ ssDNA donor strand to introduce the c.1934dupG mutation into the endogenous Asxll locus. This approach results in the insertion of a $G$ within a stretch of eigth G located in the last exon of Asxll which in turn generates a frameshift and an in-frame stop codon 
A

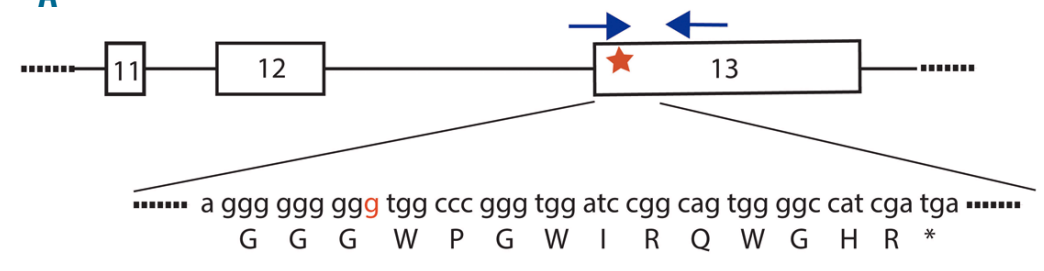

B

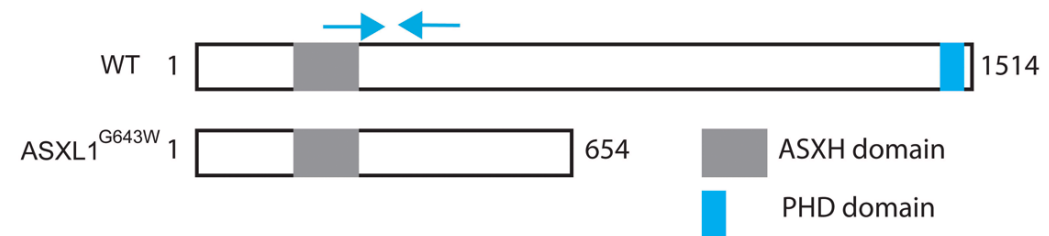

C

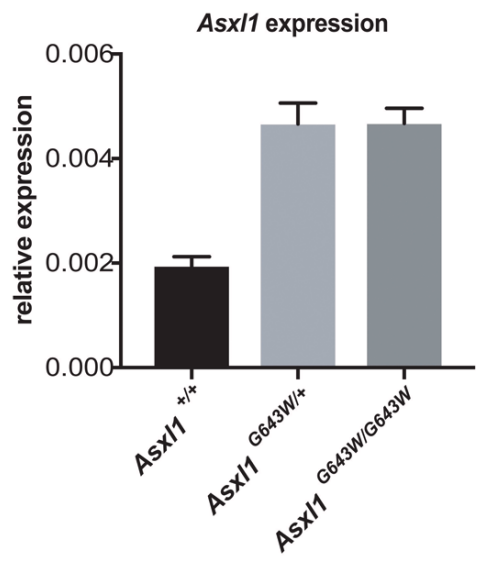

Figure 1. Generation of the $A s x / 1^{6643 W}$ mutant mouse line. (A) Schematic representation of the three last exons of the Asxl1 gene. The red star represents the G643W mutation. The " $\mathrm{g}$ " inserted in the mutated allele is indicated in red. The protein sequence is in capitals and the asterisk represents the stop codon generated as a result of the frameshift. The blue arrows represent primers used for genotyping (two forward primers have been used to selectively anneal the wild-type [WT] and mutated sequence, respectively). (B) Schematic representation of WT and G643W mutant ASX1 proteins. The major plant homeodomain (PHD) and additional sex combs homology domain (ASXH) are indicated. Amino acids are numbered. The light blue arrows represent the primers used for quantitative RT-PCR. (C) The relative expression of Asxl1 cDNA in Asxl1+/+, Asxl1 $1^{\mathrm{G} 643 \mathrm{~W} /+}$ and $A s \times 11^{\mathrm{G} 643 \mathrm{~W} / \mathrm{G643W}}$ BM cells, determined by quantitative RT-PCR ( $\mathrm{n}=3$ mice in each experimental group).

12 codons downstream, thereby precisely mimicking the human situation (Figure 1A). The mutated Asx/1 ${ }^{\mathrm{G} 643 \mathrm{~W}}$ allele expresses a truncated form of ASXL1 lacking the C-terminal plant homeodomain (Figure 1B). Both Asx/1 ${ }^{\mathrm{G} 643 \mathrm{~W} / \mathrm{+}}$ and Asxl1 1643 W/G643W mice express elevated levels of Asxl1, demonstrating that the mutated allele escapes nonsensemediated mRNA decay (Figure 1C). This is in line with the previously observed expression of truncated ASXL1 in patient cells. ${ }^{6}$ The increased levels of the mutated mRNA could potentially be the result of a feedback mechanism.

\section{The Asxl1 ${ }^{6643 W}$ variant has minimal impact on normal hematopoiesis}

We next assessed the impact of the G643W mutation in the context of normal hematopoiesis. Both $A s \times 11^{\mathrm{G} 643 \mathrm{~W} / \mathrm{t}}$ and Asx/1 ${ }^{\mathrm{G} 643 \mathrm{~W} / \mathrm{G} 643 \mathrm{~W}}$ mice were born at the expected Mendelian ratios and showed a normal lifespan. This suggests that the ASXL1 ${ }^{\mathrm{G} 643 \mathrm{~W}}$ variant has no impact on embryonic development or aging.

We next analyzed peripheral blood for the relative frequencies of the major blood lineages. Six-month old Asxl1 $1643 W /+$ and Asxl1 1643 W/G643W mice displayed no major changes within the peripheral blood compared to wildtype (WT) controls (Figure 2A). However, at 18 months, we observed a skewing towards the myeloid lineage in ASXL1 mutated mice (Figure 2B-C, Online Supplementary Figure S1A). The age-dependent skewing was accompanied by a mild splenomegaly (Figure $2 \mathrm{G}$ ).

Consistent with the lack of changes in the peripheral blood in young mice, 6-month old Asx/16643W/4 and Asx/1 $16643 / / 6643 W$ neither displayed any changes within the hematopoietic stem cell (HSC) and multipotent progenitor (MPP) compartment, nor within the distribution of mature blood lineages in the bone marrow (Figure 2D-F, Online Supplementary Figure $S 1 B$ ). Competitive transplantation of ASXL1-mutated BM cells revealed a significant but minor reduction in the ability of $A s \times 11^{\mathrm{G} 643 W / G 643 W} \mathrm{BM}$ to reconstitute hematopoiesis, suggesting that HSC functionality is only mildly affected (Figure $2 \mathrm{H}$ ).
Collectively, these findings show that mutation of ASXI1 leads to a mild and age-dependent perturbation of the hematopoietic system in mice.

\section{The ASXL1 ${ }^{\mathrm{G} 643 \mathrm{~W}}$ variant accelerates the development of CEBPA mutant acute myeloid leukemia}

Mutations in ASXL1 and CEBPA are frequently cooccurring in AML patients. ${ }^{23,24}$ In order to test the potential functional interplay between these factors, we crossed all three Asxl1 genotypes onto either a Cebpa ${ }^{f / p 30} ; \mathrm{M} \times 1 \mathrm{Cre}$ or $\mathrm{Cebpa}^{f /+}$; Mx1Cre background resulting in a total of six genotypes. We subsequently transplanted BM from these mice into lethally irradiated recipients and 3 weeks later induced the deletion of the conditional Cebpa allele by injections with polyinosinic:polycytidylic ( $\mathrm{pIpC}$ ) acid (Figure 3A). This strategy facilitates the deletion of the full-length Cebpa allele, thereby allowing the Cebpa ${ }^{p 30}$ allele to exert its oncogenic function. Consistent with previous findings, $A s \times l_{1+++}$; Cebpa $a^{\Delta p 30}$ donor cells sustain the development of AML with a median latency of 43 weeks (Figure 3B). ${ }^{26}$ Interestingly, both heterozygous and homozygous expression of the ASXL1 $1^{\mathrm{G} 643 \mathrm{~W}}$ variant significantly accelerated CEBPA mutant driven AML development, with median disease latencies of 37 and 38 weeks, respectively. In contrast, none of the control Cebpa ${ }^{N_{+}}$genotypes lead to AML, irrespective of their Asxll mutation status.

Given that Asx/1 1643 W/G643W animals, compared to their heterozygous counterparts, displayed a somewhat more pronounced phenotype during steady-state hematopoiesis, we decided to focus on this genotype in the context of CEBPA mutant AML. Whereas As $\times 11^{+++}$; Cebpa $a^{\Delta_{p 30}}$ and Asxl1 ${ }^{6643 W / G 643 W} ;$ Cebpa $a^{\Delta_{p 30}}$ leukemias appeared morphologically identical, the latter displayed a trend towards increased levels of c-Kit (Figure 3C-E, Online Supplementary Figure S2). This suggests that the Asxll mutation, at least in the context of CEBPA mutant AML, could result in a slightly more immature leukemic phenotype which would be consistent with the more aggressive nature of ASXL1 mutant AML. 
A

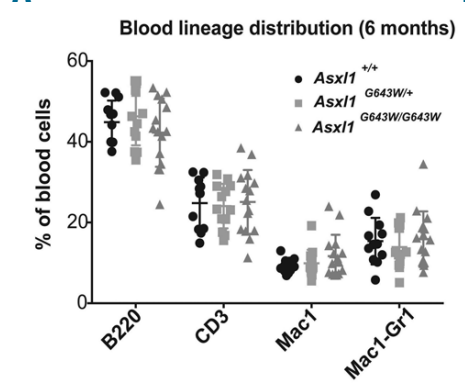

D

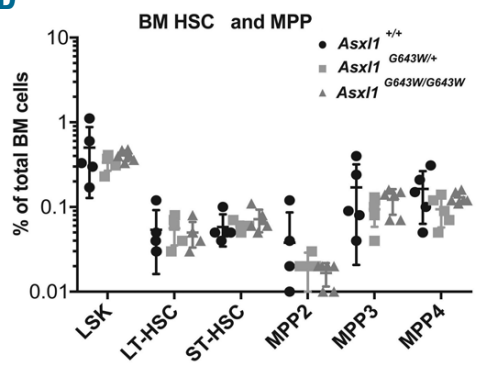

$\mathbf{F}$

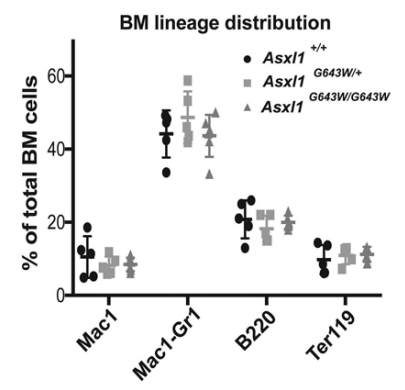

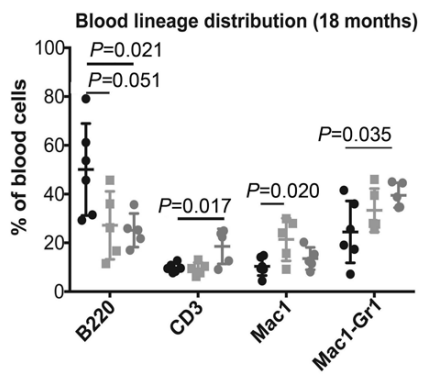

C
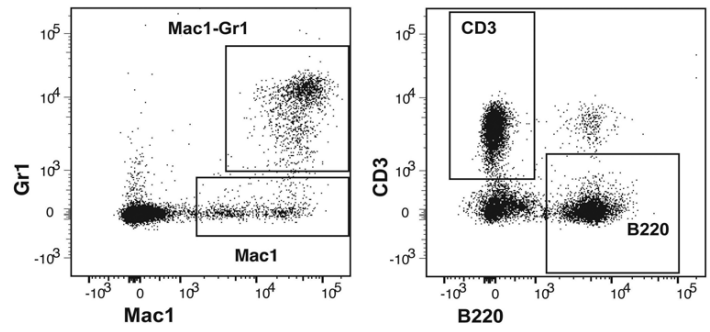

E
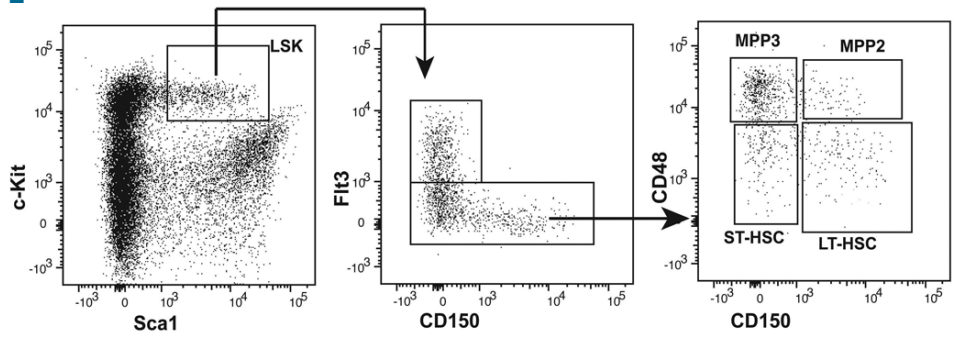

G

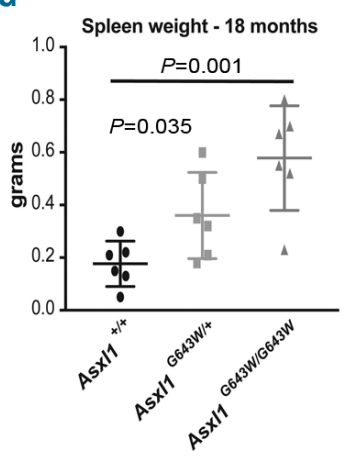

H

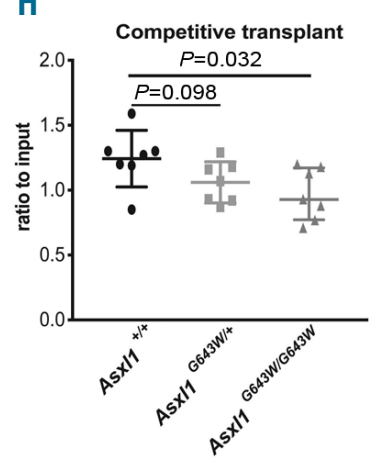

Figure 2. The ASXL1 ${ }^{\mathrm{G} 643 \mathrm{~W}}$ variant has minimal impact on normal hematopoiesis. (A) Fluorescence-activated cell sorting (FACS)-based analysis of the peripheral blood of 6-month old $A s x / 1^{+/+}, A s \times 11^{\mathrm{G} 643 \mathrm{~W} /+}$ and $A s \times 11^{\mathrm{G} 643 \mathrm{~W} / 6643 \mathrm{~W}}$ mice ( $\mathrm{n}>11$ mice in each experimental group). The relative distribution of $\mathrm{B}$ cells (B220), T cells (CD3), neutrophilic granulocytes (Mac1-Gr1) and other monocytic/granulocytic cells (Mac1) was analyzed. (B) FACS-based analysis of the peripheral blood of 18-month old Asxl1 $1^{+/+}, A s \times 11^{6643 W /+}$ and $A s \times 11^{\mathrm{G} 643 W / 6643 W}$ mice ( $\mathrm{n}>5$ mice in each experimental group). The relative distribution of $B$ cells (B220), T cells (CD3), neutrophilic granulocytes (Mac1-Gr1) and other monocytic/granulocytic cells (Mac1) was analyzed. (C) Representative FACS profiles of the data from (A-B) with the gating strategy indicated. The FACS profile represents an As $\times 11^{+/+}$control mouse. See the Online Supplementary Figure S1 for representative FACS plots of As $x / 1^{6643 W /+}$ and As $/ 1^{6643 W / 6643 W}$ animals. (D) FACS-based analysis of bone marrow hematopoietic stem cell (HSC) and multipotent progenitor (MPP) subsets in 6-month old Asxl1 ${ }^{+/+}$, Asx/1 ${ }^{6643 W /+}$ and Asxl1 $1^{\text {G643W/G643W }}$ mice ( $\mathrm{n}>5$ mice in each experimental group). (E) Representative FACS profiles of the data from (D) with the gating strategy indicated. The FACS profile represents an $A s \times 11^{+/+}$control mouse. See the Online Supplementary Figure S1 for representative FACS plots of $A s \times 11^{\mathrm{G} 643 \mathrm{~W} /+}$ and $A s \times 11^{\mathrm{G} 643 \mathrm{~W} / \mathrm{G} 643 \mathrm{~W}}$ animals. (F) Lineage distribution of mature bone marrow (BM) subsets in 6-month old $A s \times 11^{+/+}$, Asxl1 $1^{\mathrm{G} 643 \mathrm{~W} /+}$ and $A s \times 11^{\mathrm{G} 643 \mathrm{~W} / \mathrm{G} 643 \mathrm{~W}}$ mice ( $\mathrm{n}>5$ mice in each experimental group). The relative distribution of B cells (B220), T cells (CD3), neutrophilic granulocytes (Mac1-Gr1) and other monocytic/granulocytic cells (Mac1) was analyzed. (G) Spleen weights of 18-month old $A s x / 1^{+/+}, A s x / 1^{\mathrm{G} 643 W /+}$ and $A s x / 1^{\mathrm{G} 643 W / 6643 \mathrm{~W}}$ mice ( $\mathrm{n}>5$ mice in each experimental group). (H) Competitive BM transplantation of $1: 1$ mixtures of CD45.2 donor BM cells from 6-month old Asxl1 ${ }^{+/+}$, Asxl1 ${ }^{\mathrm{G643W/+}}$ or $A s \times 11^{\mathrm{G} 643 \mathrm{~W} / \mathrm{G643W}}$ mice and CD45.1 competitor cells into lethally irradiated CD45.1 recipient mice. The ratio of CD45.2 to CD45.1 in peripheral blood is depicted ( $n>7$ mice in each experimental group).

Collectively, these findings agree with the observed cooccurrence of ASXL1 and CEBPA mutations in human $\mathrm{AML}$ and suggest that these lesions co-operate in the development of AML.

\section{The ASXL1 ${ }^{\mathrm{G} 643 \mathrm{~W}}$ variant affects the expression of leukemia relevant pathways}

In order to understand the molecular underpinnings of the functional co-operation between ASXL1 and CEBPA mutations, we isolated leukemic blasts from $A s \times 11^{+/+}$; Cebpa $a^{\Delta / 30}$ and Asxl1 G643W/G643W; Cebpa $^{\Delta / p 30}$ donor-derived AML and subjected them to gene expression profiling. This analysis revealed that in the context of CEBPA mutant AML, the Asx/1 $1643 W / 6643 W$ genotype was associated with the upregulation of 177 genes and the down-regu- lation of 279 genes (adjusted $P$-value $<0.05$; Figure 4A, Online Supplementary Table S1, Online Supplementary Figure $S 3 A$ ). Interestingly, 18 of 30 of the most upregulated genes encoded pseudogenes, perhaps reflecting a role for ASXL1 in mediating their repression. Gene set enrichment analysis (GSEA) identified gene ontology processes associated with mitosis (chromosome condensation, metaphase/ anaphase transition of mitotic cell cycle and others; Figure 4B and Online Supplementary Table S2) to be up-regulated in the $A s \times 11^{\mathrm{G} 643 \mathrm{~W} / \mathrm{G} 643 \mathrm{~W}}$ genotype likely reflecting the recent finding of ASXL1 being involved in maintaining sister chromatid separation. ${ }^{27}$ Furthermore, GSEA identified gene ontology terms associated with ribosome biosynthesis (ribosome biogenesis), DNA damage (regulation of DNA damage response, signal transduction by 
A

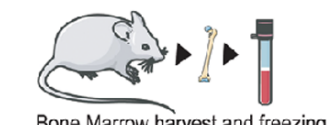

Bone Marrow harvest and freezing
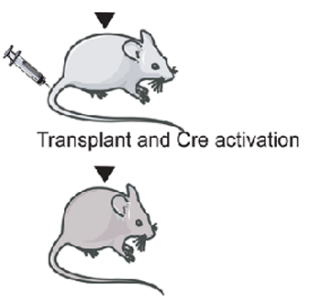

Follow disease development

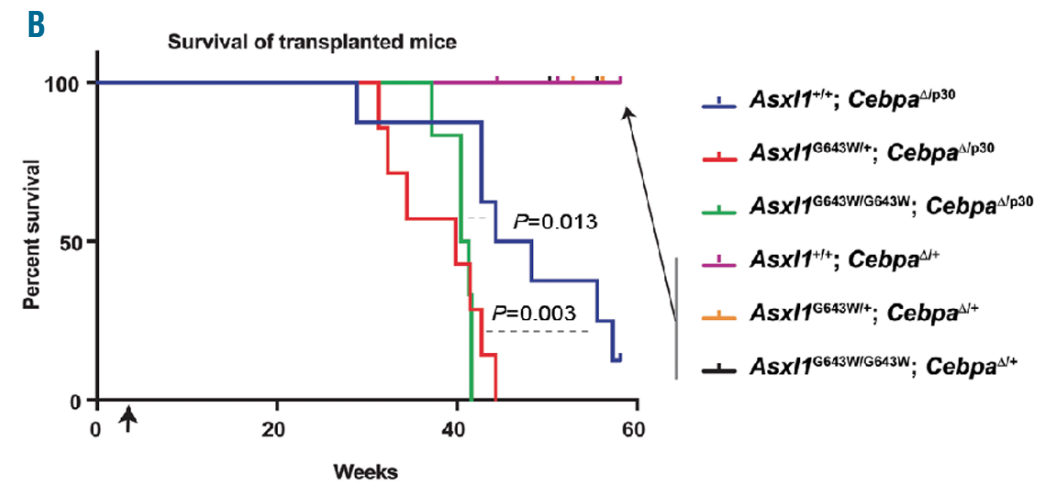

C

Normal BM

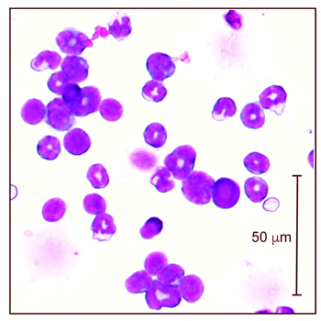

D

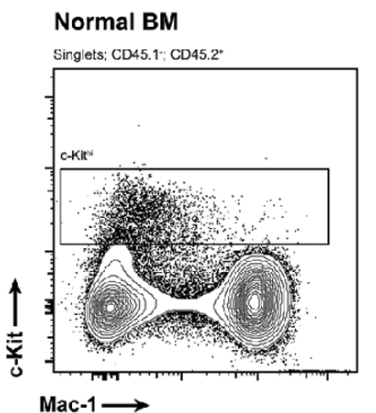

Asxl1 $1^{+/+} ;$Cebpa $^{\Delta / p 30}$

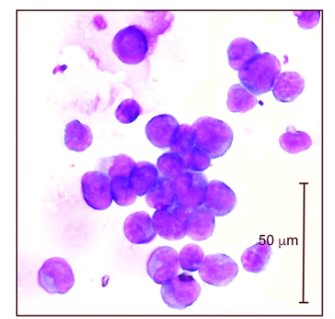

Asxl1 ${ }^{+1+} ;$ Cebpa $^{\Delta / \mathrm{p} 30}$

Singlets; CD45.1; CD45.2

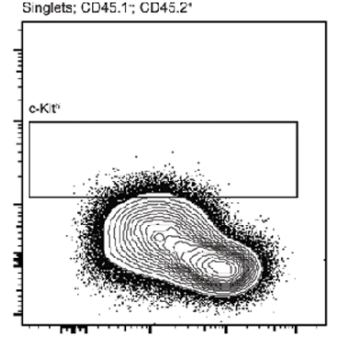

Asx/19643W/G643W; Cebpa $a^{\Delta / p 30}$

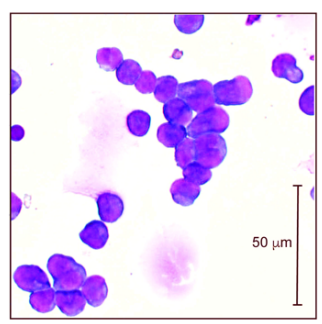

Asxl1 (G643W/G643W; Cebpa $^{\Lambda / p 30}$ Singlets; CD 45.1; CD 45.2-

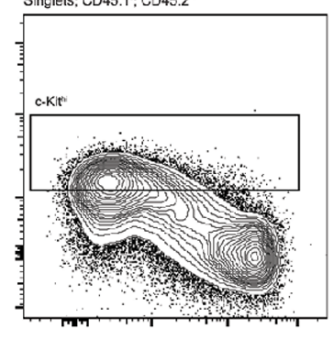

E

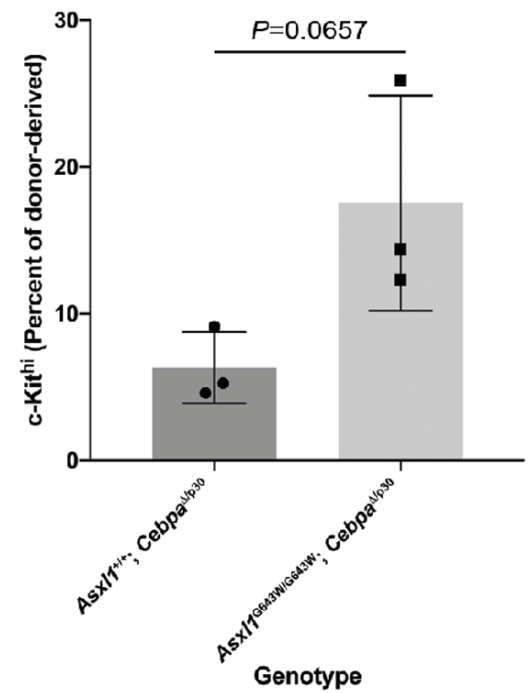

Figure 3. The ASXL1 ${ }^{\mathrm{G} 643 \mathrm{~W}}$ variant accelerate CEBPA mutant acute myeloid leukemia. (A) Schematic outline of the experiment. Briefly, bone marrow (BM) was harvested from mice with different genotypes and then transplanted into cohorts of irradiated recipients. Three weeks after the transplant, mice were injected with plpC and subsequently observed for signs of disease development over a period of 60 weeks. (B) Kaplan-Meyer survival curve of transplanted mice. The arrow indicates the time point for injection with plpC. We used a Log-rank (Mantel-Cox) test to determine statistical significance ( $\mathrm{n}>7$ mice in each experimental group). (C) Giemsa

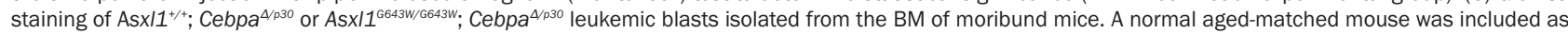

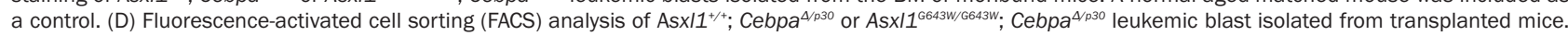
The plot shows the amount of donor-derived c-Kit positive cells. A normal aged-matched mouse was included as a control. (E) Quantification of the data from (D) ( $n=3$ mice in each experimental group).

p53 class mediator) and immune activation (antigen processing a presentation of exogenous antigen) to be downregulated in the $A s \times 11^{\mathrm{G} 643 \mathrm{~W} / \mathrm{G} 643 \mathrm{~W}}$ genotype (Figure 4C). These findings do not only point to changes in the overall metabolic status of ASXL1 mutant cells, but may also indicate a reduced activation of the immune system as well as a decreased response to genomic insults.

Focusing on individual genes, we noticed a marked upregulation of Traip in the Asx/1 $1^{\mathrm{G} 643 W / G 643 W}$ genotype (log fold change =6.7; Figure 4A, Online Supplementary Figure S3A). TRAIP is an E3 ubiquitin ligase which has been shown to be involved in the regulation of the NF- $\kappa B$ pathway, cell proliferation, regulation of the spindle assembly checkpoint, DNA replication fork recovery and more recently as a master regulator of DNA crosslink repair. ${ }^{28-32}$

Finally, in order to understand the epigenetic character- istics of the genes deregulated in the Asx/1 ${ }^{\mathrm{G} 643 \mathrm{~W} / \mathrm{G} 643 \mathrm{~W}}$ genotype, we overlaid published ChIP-seq data from Cebpa ${ }^{\Delta / 30} \mathrm{AML}^{22}$ with promoter coordinates from deregulated and constant genes, identified in our gene expression analysis described above. In these ASXL1 proficient cells, the promoters of genes that were upregulated following mutation of Asxll (in the context of CEBPA mutant AML), were characterized by low levels of "activating" histone modifications H3K4me3 and H3K27ac as well as by high level of the repressive histone mark H3K27me3 (Figure 4D, Online Supplementary Figure S3B$C)$. This combination of epigenetic marks is consistent with the low expression of these genes and previous work has demonstrated that loss of ASXL1 activity is associated with upregulation of PRC2-repressed genes. ${ }^{5,9}$ Genes that are down-regulated in the Asx/1 G643W/G643W 
A

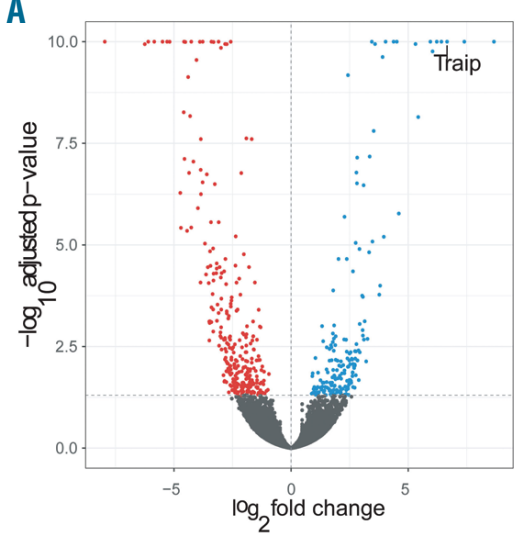

C

Ribosome biogenesis (GO:0042254)

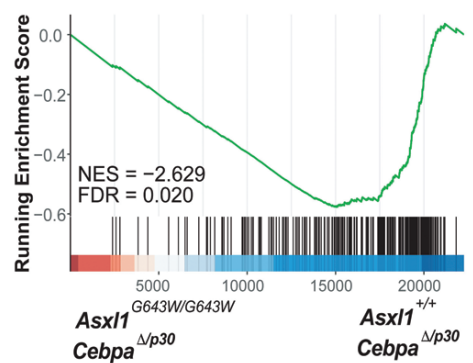

B
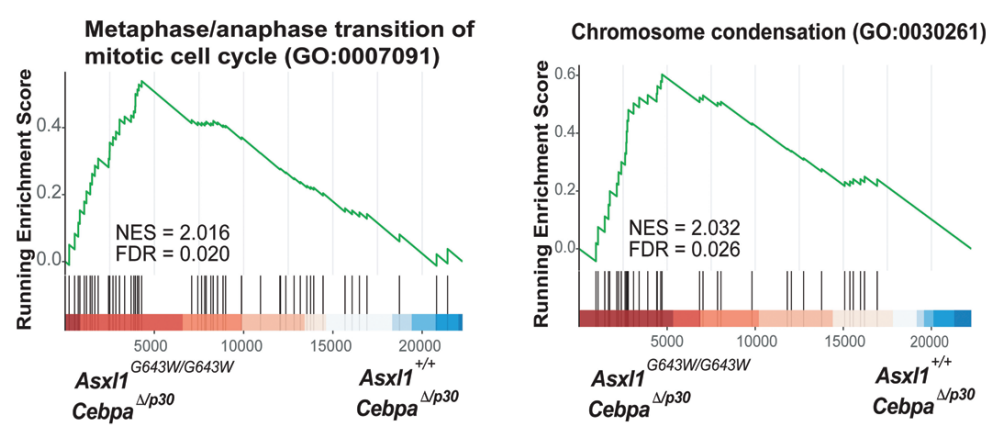

Regulation of DNA damage

response, signal transduction

by $\mathrm{p} 53$ class mediator (GO:0043516)
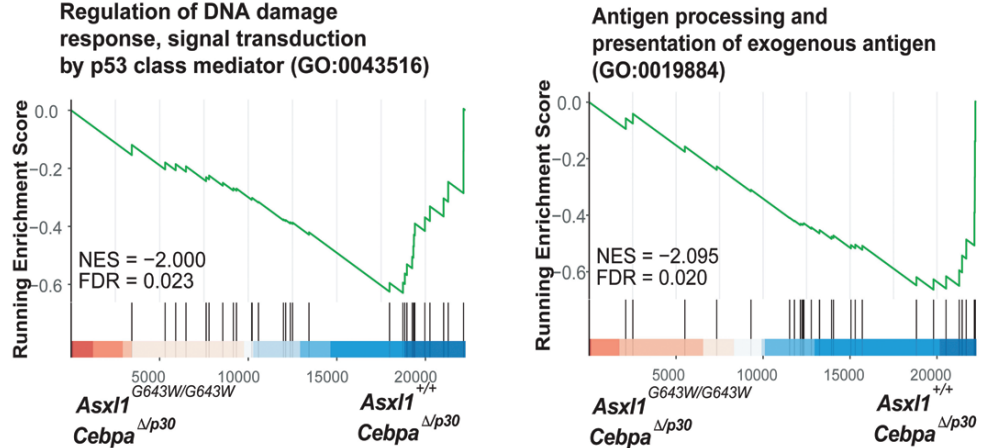

D

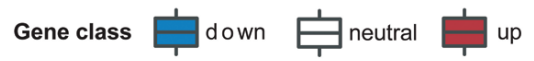
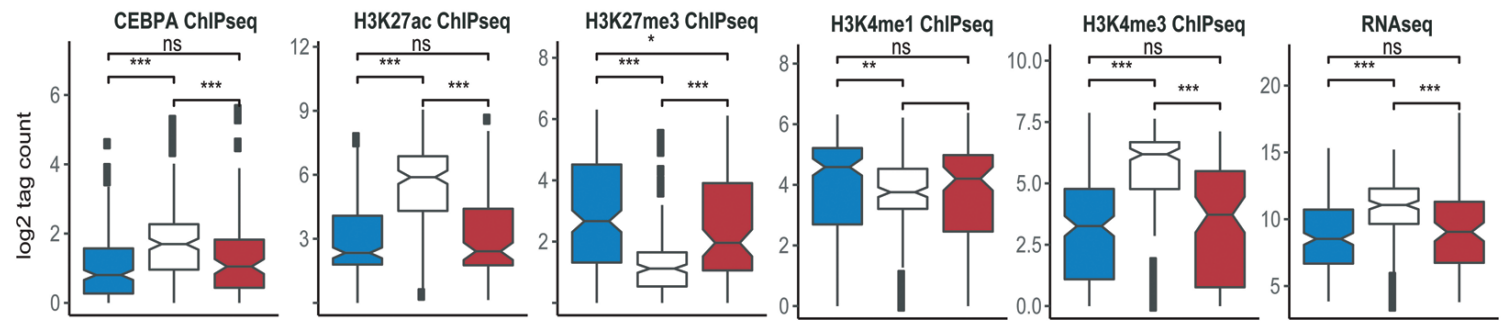

Figure 4. The ASXL1 ${ }^{\text {G643W }}$ variant affects the expression of leukemia relevant pathways. (A). Volcano plot depicting gene expression changes in AsXl1 ${ }^{6643 W / 6643 W}$

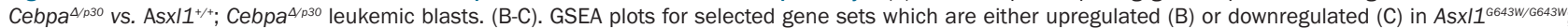
$\mathrm{Cebpa}^{4 / p 30}$ versus As $\times 11^{+/+}$; Cebpa ${ }^{4 / 030}$ leukemic blasts. (D) Boxplots showing the CHIP-signal levels of selected marks surrounding the TSS (+/- 500 bp) for genes that

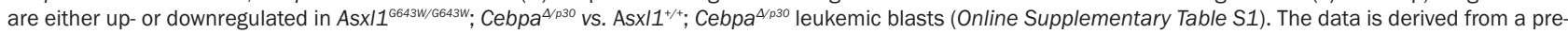
vious CEBPA mutant $\left(C_{\text {ebpa }}{ }^{p 0 / p 30}, A s \times 11\right.$ WT $)$ dataset. ${ }^{22}$ Gene expression levels as determined by RNA sequencing are also indicated. Up: upregulated genes in

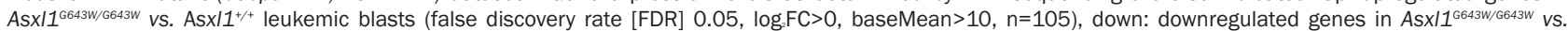
Asxl1 ${ }^{+/+}$; (FDR 0.05, $\log _{2} \mathrm{FC}<0$, baseMean $\left.>10, \mathrm{n}=201\right)$, neutral: neutral genes $(-0.01<\log \mathrm{FC}<0.01$, baseMean $>10, \mathrm{n}=165)$ in $A s \times 11^{\mathrm{G} 643 \mathrm{w} / \mathrm{G} 643 \mathrm{~W}}$ vs. Asxl1 ${ }^{+/+}$. $P$-values levels $* P<0.05 \mathrm{~s}, * * P=0.01, * * * P=0.001$ are indicated

genotype are also weakly expressed and display a similar epigenetic signature. The downregulation of these genes could potentially be due to a global decrease in H3K4me3 which has previously been found to be associated with the expression of a C-terminally truncated ASXL1 variant. ${ }^{17}$

Collectively, these data suggest that expression of the ASXL1 $1^{\mathrm{G} 643 \mathrm{~W}}$ variant affects the expression of a number of leukemic relevant genes and pathways, consistent with the role of ASXL1 as a broad epigenetic modifier.

The ASXL1 ${ }^{\mathrm{G} 643 \mathrm{~W}}$ variant is associated with increased resistance to chemotherapy in the context of CEBPA mutant acute myeloid leukemia

Failure to respond to chemotherapy is a major determi- nant of overall survival in AML and genomic stratification has associated ASXI1 mutations with adverse outcome in human AML. ${ }^{33-35}$ This raises the possibility that AML-associated mutations in ASXL1 specifically impact on the cellular response to chemotherapy. In order to test this possibility, we used our well-defined experimental set-up to assess the impact of the $A s \times 11^{\mathrm{G} 643 \mathrm{~W} / \mathrm{G} 643 \mathrm{~W}}$ genotype on the response to induction chemotherapy in the context of CEBPA mutant AML. To this end, we transplanted Asxl1 $1^{++}$; Cebpa $a^{\Delta / 30}$ and Asxl1 ${ }^{\text {G643W/G643W }}$; Cebpa $a^{\Delta / 30}$ secondary AML (a total of seven clones) into recipient mice and subjected them to low-dose induction chemotherapy 3 weeks post transplantation (Figure 5A, Online Supplementary Figure S4). Compared to the ASXL1 ${ }^{\mathrm{WT}}$ cohort, ASXL1 ${ }^{\mathrm{G} 643 \mathrm{~W}}$ mice displayed reduced response to chemotherapy as 
A

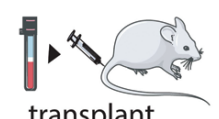

transplant

days 1

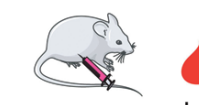

Chemo-treatment blood analysis Follow disease development
C

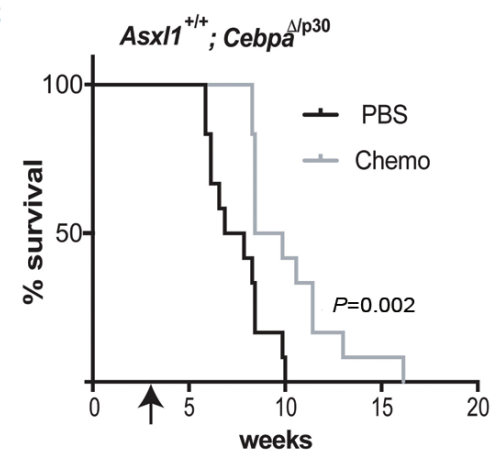

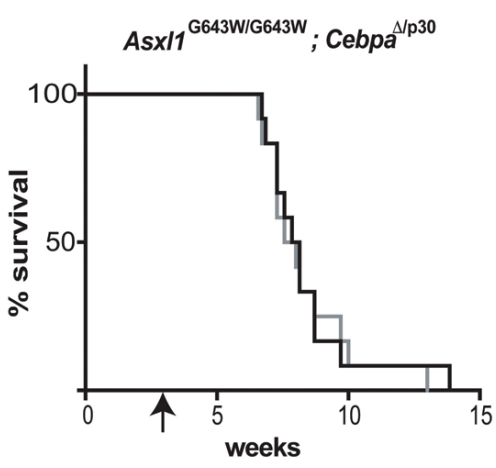

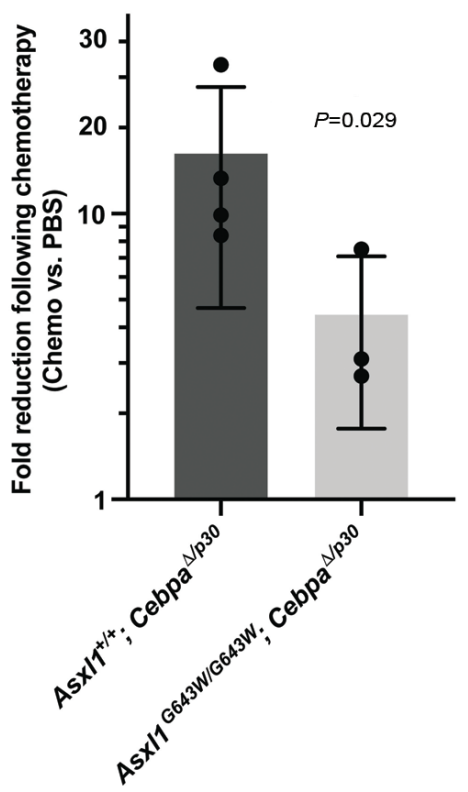

Figure 5. The ASXL1 ${ }^{\text {G643w }}$ variant is associated with increased resistance to chemotherapy. (A) Schematic outline of the chemotherapy treatment set-up. Briefly, sublethally irradiated recipient mice were transplanted with bone marrow (BM) cells harvested from leukemic mice. Twenty-one days after transplant, recipient mice were treated with standard induction chemotherapy for 5 days. Three days later, blood was harvested for analysis and leukemic mice were subsequently monitored for disease development. (B) Analysis of peripheral leukemic numbers in mice after chemotherapy/vehicle treatment. Each data point represents the fold change difference between the vehicle-treated and chemotherapy-treated groups for a given clone ( $n=12$ mice per clone). The two different genotypes, Asx/1 ${ }^{+/+} ;$Cebpa ${ }^{4 / p 30}$ and Asxl1G643W/G643W Cebpa ${ }^{4 / p 30}$, were represented by four and three clones, respectively. The responses of individual clones are indicated in the Online Supplementary Figure S4. A one-tailed Mann-Whitney U test was used to assess statistical significance. (C) Kaplan-Meyer survival curves of leukemic mice after chemotherapy/vehicle treatment. The data represent the aggregate of two different leukemic clones ( $n=6$ recipients of each clone, clones 1-2 and 5-6) for each of the two different genotypes (Asxl1 $1^{++} ;$Cebpa $^{4 / p 30}$ and AsxI1G643W/G643W; Cebpa ${ }^{4 / p 30}$ ).

measured by the decrease of leukemic cells in the peripheral blood 3 days post-treatment cessation (Figure 5B). This translated into an increased latency for the treated ASXL1 ${ }^{\text {WT }}$ cohort whereas chemotherapy had no impact on survival of the ASXL1 ${ }^{\mathrm{G} 643 \mathrm{~W}}$ mutant cohort (Figure $5 \mathrm{C}$ ).

Taken together, these findings demonstrate that mutation of ASXI1 renders CEBPA mutated AML largely resistant to chemotherapy.

\section{Discussion}

Myeloid diseases such as AML are developing from premalignant clones which mostly harbor lesions in epigenetic regulators. ${ }^{3,4}$ One of these regulators is ASXL1 which is consequently frequently mutated in both MDS and $\mathrm{AML}$ as well as in premalignant settings such as CHIP. This mutational profile raises the question whether ASXL1 plays a functional role in full-blown AML or whether it merely provides a fertile ground in which AML can evolve.

There has been considerable confusion concerning the molecular mechanisms by which ASXL1 mutations sustain AML development or hematological deficiencies, specifically whether ASXI1 mutations act as dominant negatives. Some of this confusion is related to the inherent difficulties in detecting ASXL1 by western blotting (issues which we also experienced) thus making it nearly impossible to determine whether the mutated protein is present or not. However, overexpression of ASXL1 variants was associated with hematological malignancies raising the possibility that the mutated protein could act as a domi- nant negative. ${ }^{6,14,15}$ Our analysis of heterozygous and homozygous $A s \times 11^{\mathrm{G} 643 \mathrm{~W}}$ mice in the context of normal hematopoiesis suggests that the $A s \times 11^{\mathrm{G} 643 \mathrm{~W}}$ variant has a dose-dependent impact in this context. Given that complete loss of Asxll leads to more pronounced hematopoietic phenotypes, the most restrained explanation from the in vivo work is that the ASXL1 $1^{\mathrm{G} 643 \mathrm{~W}}$ variant is hypomorphic or, alternatively, that it exerts a combination of dominant negative and hypomorphic effects. ${ }^{10-12}$

Expression of the Asxl1 ${ }^{\mathrm{G} 643 \mathrm{~W}}$ variant in the context of CEBPA mutant AML significantly accelerated AML development and was associated with marked resistance to induction chemotherapy. These findings are not only in perfect alignment with the co-occurrence of CEBPA and ASXL1 lesions in human AML, but also with the overall poor prognosis of ASXL1 mutated human AML. Hence the resistance towards chemotherapy is likely underlying the poor prognosis of AXI1 mutated AML. ${ }^{33-35}$ It would be interesting to test if other mouse models can recapitulate this behavior beyond CEBPA mutant AML.

Interestingly, gene expression analysis demonstrated that Asxl1 1 G643W/G643W; Cebpa ${ }^{\Delta / 30}$ exhibited downregulation of signatures associated with immune activation perhaps reflecting that the Asxll lesion renders the developing leukemia less visible to the immune system. We also observed a reduction in activation of the DNA damage pathways in ASXL1 mutated cells which could potentially reflect efficient clearing of ongoing genomic insults. Here our finding of the marked upregulation of Traip is of particular interest as the corresponding protein has recently been identified as a master regulator of DNA crosslink repair. ${ }^{32}$ Thus, our data strongly suggest that Asxl1 lesions have functional conse- 
quences in the context of CEBPA mutant AML and that they therefore provide more than a fertile ground for AML development.

To summarize, we have generated a novel ASXL1 ${ }^{\mathrm{G} 643 \mathrm{~W}}$ mouse model mimicking the most commonly observed ASXL1 lesion mutation in human MDS and AML patients. Consistent with the co-occurrence of CEBPA and ASXL1 lesions in AML, the ASXL1 ${ }^{\mathrm{G} 643 \mathrm{~W}}$ variant accelerates AML development in the context of CEBPA mutant AML. Finally, the observed resistance towards chemotherapy conferred by the ASXL1 $1^{\mathrm{G} 643 \mathrm{~W}}$ variant provides us with an experimental handle for future experiments aimed at its reversal.

\section{Disclosures}

No conflicts of interest to disclose

\section{Contributions}

$T D, M B S, A M H$ and $A S W$ carried out the experiments; TD,
$A W, A K, S P$ and BTP analyzed data; TDA and BP drafted the manuscript which was proofread by all authors; BTP directed the research.

\section{Acknowledgments}

We thank Javier Martin Gonzalez and the Transgenic Core Facility for the generation of the mutant strain.

\section{Funding}

This work was supported by a grant from the Danish Cancer Society (to Teresa D'Altri) and through a center grant from the Novo Nordisk Foundation (Novo Nordisk Foundation Center for Stem Cell Biology, DanStem; Grant Number NNF17CC0027852). The present work is also part of the Danish Research Center for Precision Medicine in Blood Cancers funded by the Danish Cancer Society grant no. R223-A13071 and Greater Copenhagen Health Science Partners.

\section{References}

1. Gelsi-Boyer V, Trouplin V, Adelaide J, et al. Mutations of polycomb-associated gene ASXL1 in myelodysplastic syndromes and chronic myelomonocytic leukaemia. Br J Haematol. 2009;145(6):788-800

2. Bejar R, Stevenson K, Abdel-Wahab O, et al. Clinical effect of point mutations in myelodysplastic syndromes. N Engl J Med. 2011;364(26):2496-2506.

3. Jaiswal S, Fontanillas P, Flannick J, et al. Agerelated clonal hematopoiesis associated with adverse outcomes. N Engl J Med. 2014; 371(26):2488-2498

4. Genovese G, Kahler AK, Handsaker RE, et al. Clonal hematopoiesis and blood-cancer risk inferred from blood DNA sequence. $\mathrm{N}$ Engl J Med. 2014;371(26):2477-2487.

5. Abdel-Wahab O, Adli M, LaFave LM, et al. ASXL1 mutations promote myeloid transformation through loss of PRC2-mediated gene repression. Cancer Cell. 2012; 22(2):180-193.

6. Inoue $D$, Matsumoto $M$, Nagase $R$, et al. Truncation mutants of ASXL1 observed in myeloid malignancies are expressed at detectable protein levels. Exp Hematol. 2016;44(3):172-176.1

7. Dey A, Seshasayee D, Noubade R, et al. Loss of the tumor suppressor BAP1 causes myeloid transformation. Science. 2012; 337(6101):1541-1546.

8. Scheuermann JC, de Ayala Alonso AG, Oktaba K, et al. Histone H2A deubiquitinase activity of the Polycomb repressive complex PR-DUB. Nature. 2010;465(7295):243-247.

9. Abdel-Wahab O, Dey A. The ASXL-BAP1 axis: new factors in myelopoiesis, cancer and epigenetics. Leukemia. 2013;27(1):1015

10. Abdel-Wahab O, Gao J, Adli M, et al. Deletion of Asxl1 results in myelodysplasia and severe developmental defects in vivo. J Exp Med. 2013:210(12):2641-2659.

11. Fisher CL, Pineault N, Brookes C, et al. Lossof-function additional sex combs like 1 mutations disrupt hematopoiesis but do not cause severe myelodysplasia or leukemia. Blood. 2010;115(1):38-46

12. Wang J, Li Z, He Y, et al. Loss of Asxl1 leads to myelodysplastic syndrome-like disease in mice. Blood. 2014;123(4):541-553.

13. Asada S, Fujino T, Goyama S, Kitamura T.
The role of ASXL1 in hematopoiesis and myeloid malignancies. Cell Mol Life Sci. 2019;76(13):2511-2523.

14. Asada S, Goyama S, Inoue D, et al. Mutant ASXI1 cooperates with BAP1 to promote myeloid leukaemogenesis. Nat Commun. 2018:9(1):2733

15. Balasubramani A, Larjo A, Bassein JA, et al. Cancer-associated ASXL1 mutations may act as gain-of-function mutations of the ASXL1-BAP1 complex. Nat Commun. 2015; 6:7307.

16. Hsu YC, Chiu YC, Lin CC, et al. The distinct biological implications of Asxl1 mutation and its roles in leukemogenesis revealed by a knock-in mouse model. J Hematol Oncol. 2017;10(1):139.

17. Nagase $\mathrm{R}$, Inoue $\mathrm{D}$, Pastore $\mathrm{A}$, et al. Expression of mutant Asxl1 perturbs hematopoiesis and promotes susceptibility to leukemic transformation. J Exp Med. 2018:215(6):1729-1747.

18. Pabst T, Mueller BU, Zhang P, et al. Dominant-negative mutations of CEBPA, encoding CCAAT/enhancer binding protein-alpha (C/EBPalpha), in acute myeloid leukemia. Nat Genet. 2001;27(3):263-270.

19. Papaemmanuil E, Gerstung M, Bullinger L, et al. Genomic classification and prognosis in acute myeloid leukemia. N Engl J Med. 2016:374(23):2209-2221.

20. Ohlsson E, Schuster MB, Hasemann M Porse BT. The multifaceted functions of C/EBPalpha in normal and malignant haematopoiesis. Leukemia. 2016;30(4):767775.

21. Kirstetter P, Schuster MB, Bereshchenko O, et al. Modeling of C/EBPalpha mutant acute myeloid leukemia reveals a common expression signature of committed myeloid leukemia-initiating cells. Cancer Cell. 2008;13(4):299-310.

22. Jakobsen IS, Laursen LG, Schuster MB, et al. Mutant CEBPA directly drives the expression of the targetable tumor-promoting factor CD73 in AML. Sci Adv. 2019; 5(7):eaaw4304

23. Rose D, Haferlach T, Schnittger $S$, Perglerova K, Kern W, Haferlach C. Subtypespecific patterns of molecular mutations in acute myeloid leukemia. Leukemia. 2017; 31(1):11-17

24. Su L, Tan Y, Lin $\mathrm{H}$, et al. Mutational spectrum of acute myeloid leukemia patients with double CEBPA mutations based on next-generation sequencing and its prognostic significance. Oncotarget. 2018, 9(38):24970-24979.

25. Chou WC, Huang $\mathrm{HH}$, Hou $\mathrm{HA}$, et al Distinct clinical and biological features of de novo acute myeloid leukemia with additional sex comb-like 1 (ASXL1) mutations. Blood. 2010;116(20):4086-4094.

26. Schuster MB, Frank A-K, Bagger FO, Rapin N, Vikesaa J, Porse BT. Lack of the p42 form of $\mathrm{C} / \mathrm{EBP} \alpha$ leads to spontaneous immortalization and lineage infidelity of committed myeloid progenitors. Exp Hematol. 2013; 41(10):882-893.e16.

27. Li Z, Zhang P, Yan A, et al. ASXL1 interacts with the cohesin complex to maintain chromatid separation and gene expression for normal hematopoiesis. Sci Adv. 2017;3(1):e1601602

28. Chapard C, Hohl D, Huber M. The role of the TRAF-interacting protein in proliferation and differentiation. Exp Dermatol. 2012; 21(5):321-326.

29. Chapard C, Meraldi P, Gleich T, Bachmann D, Hohl D, Huber M. TRAIP is a regulator of the spindle assembly checkpoint. J Cell Sci. 2014;127(Pt 24):5149-5156.

30. Feng W, Guo Y, Huang J, Deng Y, Zang J, Huen MS. TRAIP regulates replication fork recovery and progression via PCNA. Cel Discov. 2016;2:16016.

31. Hoffmann S, Smedegaard S, Nakamura K, et al. TRAIP is a PCNA-binding ubiquitin ligase that protects genome stability after replication stress. J Cell Biol. 2016;212(1):63-75

32. Wu RA, Semlow DR, Kamimae-Lanning AN, et al. TRAIP is a master regulator of DNA interstrand crosslink repair. Nature. 2019;567(7747):267-272.

33. Schnittger S, Eder C, Jeromin S, et al. ASXL1 exon 12 mutations are frequent in AML with intermediate risk karyotype and are independently associated with an adverse outcome. Leukemia. 2013;27(1):82-91.

34. Paschka P, Schlenk RF, Gaidzik VI, et al ASXL1 mutations in younger adult patients with acute myeloid leukemia: a study by the German-Austrian Acute Myeloid Leukemia Study Group. Haematologica. 2015; 100(3):324-330.

35. Pratcorona M, Abbas S, Sanders MA, et al Acquired mutations in ASXL1 in acute myeloid leukemia: prevalence and prognostic value. Haematologica. 2012;97(3):388 392. 